\title{
A study of strain and deformation measurement using the Arduino microcontroller and strain gauges devices
}

\author{
Anderson Langone Silva ${ }^{10}$, Marcus Varanis ${ }^{*}\left[{ }^{0}\right.$, Arthur Guilherme Mereles $^{1}$, Clivaldo Oliveira ${ }^{1}$, \\ José Manoel Balthazar² \\ ${ }^{1}$ Universidade Federal da Grande Dourados, Faculdade de Engenharia, Dourados, MS, Brasil \\ ${ }^{2}$ Universidade Tecnológica Federal do Paraná, Departamento de Eletrônica, Campus Ponta Grossa, PR, Brasil
}

Received on July 09, 2018; Revised on October 09, 2018; Accepted on November 06, 2018.

\begin{abstract}
This paper presents a methodology for the measurement of strain by means of strain-gauges, where the data acquisition is done by using an Arduino Uno board, due to the low-cost and the easy manipulation of this microcontrollers. The measured signal conditioning is performed by means of a Wheatstone Bridge and then discretized by an Analog-Digital Converter (ADC) external to the Arduino. For the validation of the proposed measurement system, experiments are performed on a cantilever beam and on a cantilever-supported shaft, where the experimental results are compared with those obtained analytically and by simulation using the finite element method. The results obtained are in agreement with the literature and demonstrate that the proposed system has satisfactory accuracy.
\end{abstract}

Keywords: Strain gauge, Arduino, Low-cost data acquisition

\section{Introduction}

In many practical situations in engineering, the knowledge of the stresses and strains that are applied in a structure is of vital importance, since the predictability of the materials depend closely on these parameters. Besides, it is important to avoid deformations so large that they may prevent the structure from fulfilling the purpose for which it was intended [1]. Generally, mathematical models or finite elements methods (FEM) are needed to predict the stresses in a structure. There are some situations, however, were the modeling is not possible or its results are rough approximations that do not agree with the real loads acting on the structure. In these cases, in order to avoid overdimensioning, the experimentation is the way around.

There are many experimental procedures aimed at measuring mechanical strain, such as using strain-gauges, which can be optical [8] or mechanical [9]; or using electrical strain gauges, that can be based on resistive [12], capacitive [4], inductive [3] and photoelectric [10]. One of the most common applied method is based on the use of resistive-based strain-gauges. These devices allow the strain to be measured directly by measuring the change in the electrical resistance of them as they are subjected to deformations, which is commonly done by using a Wheatstone bridge. The gauges can also be used to measure forces and torques acting on a structure, as these quantities are directly related to strain. However, since

*Correspondence email address: marcusvaranis@ufgd.edu.br the strain-gauges are expensive and not reusable, the overall cost of the experimental procedure is generally high.

In such cases, the use of the Arduino microcontroller together with low-cost sensors is encouraged. This use brings a lot of benefits such as the easy implementation and low overall cost of the apparatus. For some examples, in [5] a low-cost Arduino-based wire strain-gauge is proposed for earth flow/landslide monitoring, where a prototype was built with an Arduino Uno board, a data logging RTC and a operating temperature sensor; the field tests showed high reliability of the experimental apparatus. In [7] an ultra-light strain-gauge device for the assessment of the mechanical properties of the human skin in vivo is presented, where the Arduino Mega 2560 was used to acquire the dada from the sensors. Some other applications are presented in 6], [2] and [13].

This paper presents a methodology for stress and strain measurement using strain-gauges and the Arduino microcontroller with the objective of being used on experimental analysis in solid mechanics disciplines. The equipment normally used for this purpose has a high-cost and the procedures to manipulate them can be complex. In this manner, in this paper a methodology for strain measurement using devices of low-cost and easy-use is proposed, allowing its use as an education tool in undergraduate courses. For the evaluation of the proposed methodology, tests were performed in two different mechanical systems and the results obtained were compared with the analytical ones. 
This work consists of some sections that are described as follows. In the section 2 the mathematical models used to obtain the analytical results are presented. In the subsection 2.1 the deformation model for the cantilever beam is presented and in the subsection 2.2 the model for a crimped-supported shaft is shown. In the section 3 the configuration used in the experiment is presented, in the subsection 3.1 the strain gauge is presented, in the subsection 3.2 the operation and the possible configurations of the Wheatstone bridge is presented, and in the section 3.3 the circuit of the proposed data acquisition system and its components is discussed. In the section 4 the experimental procedures used to perform the tests are discussed and the section ref results discusses the results obtained. In the section 6, some final considerations are made.

\section{Analytical models}

In this section, the analytical models used to perform the comparison with the experimental results are discussed. In the subsection 2.1 the mathematical model used to obtain the analytical deformation of the cantilever beam under bending is presented. In the subsection 2.2, it is presented the mathematical model used to obtain the analytical deformation of a crimped-supported shaft. The stress and strain models used in this paper are widely explored in the literature, both analytical methods 1, 14, 16 and methods based on finite elements 17, 19.

\subsection{Cantilever beam}

For the experiment of the clamped beam at one end and free at the other we have the following expression for the tension at the surface of the beam, $1,14,16$

$$
\sigma=\frac{M \rho}{I}
$$

where $M$ is the resulting bending moment, $I$ is the moment of inertia of the cross-sectional area, and rho is the distance from the neutral line to the point of interest. The moment of inertia is given by

$$
I=\frac{b h^{3}}{12}
$$

where $b$ is the width of the beam and $h$ is its thickness. Substituting Equation (2) into Equation (1) and $c$ for $h / 2$ we have

$$
\sigma_{\max }=\frac{6 M}{b h^{2}}
$$

which corresponds to the tension $\sigma_{\max }$ on the surface of the beam when subjected to a bending moment $M$. It is known that the deformation $\epsilon$ can be obtained by means of the Equation 4. known as the Hooke's Law, where $E$ is the modulus of elasticity of the material.

$$
\epsilon=E \sigma
$$

Substituting Equation 3 into Equation 4 gives the equation for the deformation at the beam surface when subjected to bending, Equation 5

$$
\epsilon=E \frac{6 M}{b h^{2}}
$$

\subsection{Crimped-supported shaft}

It is known that the maximum shear stress $\tau_{\max }$ on the surface of an axis with radius $c$ subjected to a torque with intensity $T$ is given by $1,14,16$

$$
\tau_{\max }=\frac{T c}{J}
$$

where $J$ is the polar moment of inertia of the shaft section, in this case given by

$$
J=\frac{\pi c^{4}}{2}
$$

Replacing the equation 7 in the equation 6 gives the equation for the case under analysis.

$$
\tau_{\max }=\frac{2 T}{\pi c^{3}}
$$

In order to obtain the shear deformation, the Hooke's Law for torsion is applied, according to the equation 9 , in the Equation 8

$$
\begin{gathered}
\tau=G \gamma \\
\gamma=\frac{2 T G}{\pi c^{3}}
\end{gathered}
$$

In equations 10 and 9 the constant $G$ corresponds to the shear modulus of the material.

Um estudo aprofundado sobre o assunto pode ser visto em [CITAR POPOV, HARDOG E TIMOSHENKO]. POPOV, Egor Paul; BALAN, Toader A. Engineering mechanics of solids. Englewood Cliffs, NJ: Prentice Hall, 1990. DEN HARTOG, Jacob Pieter. Advanced strength of materials. Courier Corporation, 2014.

\section{Experimental setup}

In this section the experimental aspects of the work are discussed. The subsection 3.1 treats strain gauges, the subsection 3.2 presents the Wheatstone bridge and in the subsection 3.3 the data acquisition system and the devices used are presented.

\subsection{Strain gauge}

Strain gauges are sensors based on electrical resistance, which generate a variation in its electrical resistance when subjected to a deformation in its length. They are composed of a metal grid glued to a thin base. When attached to a body under deformation the strain gauge undergoes the same deformation, in this way the grids 
increase its size, consequently generating a variation in the initial resistance of the strain gauge, being the variation of the resistance with the deformation linear. It is possible to find strain-gauges of electrical resistance in the most diverse formats, but in this work only the general purpose strain-gauge for uniaxial use are used. In Figure 1, two common formats of strain-gauges are shown. The Figure $1 \mathrm{a}$ shows an uniaxial general purpose straingauge, which is the most used model in deformation instrumentation in everyday applications. In the Figure $1 \mathrm{~b}$ it is shown a triaxial rosette-type strain-gauge, used when it is necessary to know the stress state of the test body and it consists of three uniaxial strain-gauges.

An important factor of strain gauges is their sensitivity to deformation, commonly called as gauge factor, $G F$. This factor is given by the relationship between the resistance variation and the deformation, as shown in Equation 11. By manipulating the equation 11 it is possible to obtain an equation for the strain as a function of the variation of the resistance of the strain-gauge, Equation ref strain. In equations 11 and ref strain, $K$ is the gauge factor, $R_{0}$ is the nominal resistance of the strain-gauge, $\Delta$ is its variation, and $\epsilon$ is the strain that the strain gauge was submitted $[20,21]$.

$$
\begin{gathered}
K=\frac{\Delta R_{s} / R_{s}}{\epsilon} \\
\frac{\Delta R_{s}}{R_{s}}=K \epsilon
\end{gathered}
$$

For strain analysis in the proposed systems, straingauges model BF350-3AA were utilized, which have nominal resistance of 350 ohms and gauge factor of 2 .

\subsection{Wheatstone bridge}

In resistive sensors in which only small variations of resistance occur, it is necessary to use auxiliary circuits for their instrumentation, in order to allow a good sensitivity to the measuring instrument. In the case of deformation

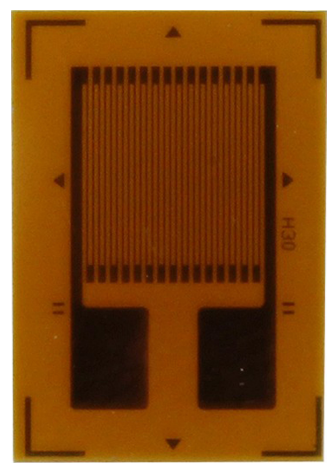

(a)

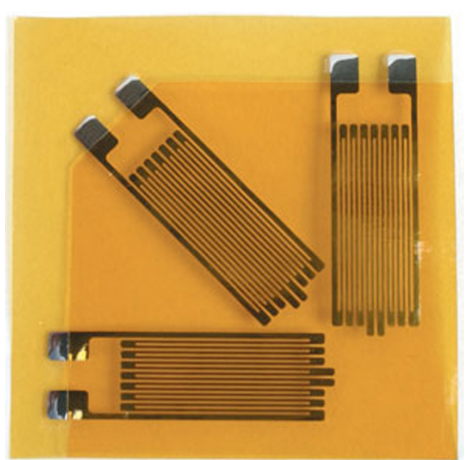

(b)
Figure 1: Two common strain gauge formats: 1a general purpose uniaxial strain gauge and $1 \mathrm{~b}$ triaxial rosette. instrumentation, where the resistance variations of the strain-gauges are minimal, the Wheatstone bridge circuit is used. This circuit allows one to measure the resistance of unknown resistors through the balance of the circuit, allowing to carry out measurements with great precision. The generic circuit of the Wheatstone Bridge is shown in Figure 2 where $R_{1}, R_{2}, R_{3}$ and $R_{4}$ are resistors, $V_{e x}$ is a power supply of direct current and $V$ is a voltmeter. Applying the Kirchhoff's first law, a relationship between the supply voltage $V_{e x}$, the voltage read by the voltmeter $V$ and the values of the resistors is obtained, Equation 13. 20,21.

$$
V=\left(\frac{R_{3}}{R_{3}+R_{4}}-\frac{R_{2}}{R_{1}+R_{2}}\right) V_{e x}
$$

The Wheatstone Bridge circuit can be a quarter, half or full bridge type, depending on the number of transducers and the required accuracy. Its principle of operation is based on the voltage equilibrium between the two arms of the circuit. In order to have voltage equilibrium, $V=0$, the relation in Equation 14 must be satisfied.

$$
\frac{R_{1}}{R_{2}}=\frac{R_{4}}{R_{3}}
$$

In the quarter bridge circuit only an active strain gauge is used, so in the circuit, the resistor $R_{4}$ is replaced by the strain gauge $S_{4}$, as in the Figure 3 a so that the resistors $R_{1}, R_{2}$ and $R_{3}$ are passive resistors with fixed resistance values. In order to maintain the output of the circuit null, the resistor $R_{1}$ can be chosen to have the same resistance as $S_{1}$, while $R_{2}$ and $R_{3}$ can be equal, in this way the equilibrium of Equation 14 is obtained. Although this circuit is easy to assemble due to the existence of only

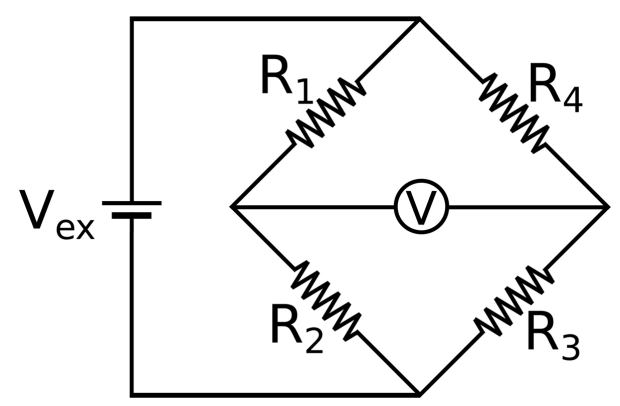

Figure 2: Wheatstone bridge standard circuit.

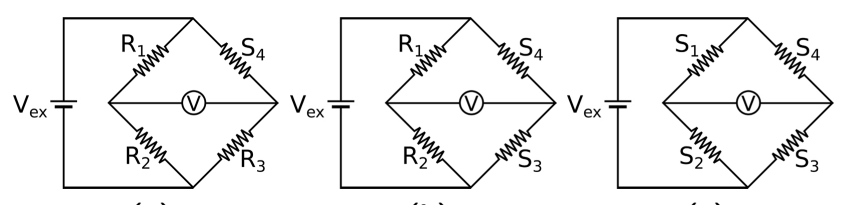

(a)

(b)

(c)

Figure 3: Common Wheatstone bridge configurations, circuits of: $3 a$ 1/ 4 bridge, $3 \mathrm{~b} 1 / 2$ bridge and $3 \mathrm{c}$ complete bridge. 
one strain gauge, the measurements are susceptible to inacurracies due to temperature.

Considering this case under analysis, taking the equation 13 considering all resistors with resistance $R$ and the strain-gauge $S_{1}$ with resistance equal to $R \pm \Delta R$ where $+\Delta R$ corresponds to traction, $+\epsilon$, and $-\Delta R$ corresponds to compression, $-\epsilon$, we get the equation 15

$$
V=\left(\frac{R}{2 R+\Delta R}-\frac{1}{2}\right) V_{e x}
$$

Manipulating the equation 15 and dividing the numerator and denominator by $R$, the equation 16 is obtained, where the term $\Delta R / R$ can be replaced according to Equation 12 obtaining the equation 18 .

$$
\begin{gathered}
V=\frac{\frac{\Delta R}{R}}{4+\frac{\Delta R}{R}} V_{e x} \\
\frac{V}{V_{e x}}=\frac{K \epsilon}{4+K \epsilon}
\end{gathered}
$$

In the equation 17 the ratio of the voltages, $V / V_{e x}$ has units of $[V / V]$, for convenience is usually multiplied by a factor of 1000 with the purpose of the unit of the ratio between the voltages to be $[m V / V]$, also for convenience the equation is multiplied by $10^{-6}$ thus the deformation $\epsilon$ pass to be microstrain, $[\mu m / m]$, obtaining the equation 18

$$
\frac{V}{V_{e x}}=\frac{K \epsilon \times 10^{-3}}{4+K \epsilon \times 10^{-6}}
$$

In the half-bridge circuit there are two active straingauges, so that the resistors $R_{3}$ and $R_{4}$ are replaced by the strain-gauges $S_{3}$ and $S_{4}$, respectively, as in Figure $3 \mathrm{~b}$ In this case it is common for the strain-gauges to be positioned so that while $S_{3}$ is subject to a deformation $-\epsilon$, $S_{4}$ is subject to a $+\epsilon$ deformation. In order to maintain the equilibrium of the circuit, the resistors $R_{1}$ and $R_{2}$ are chosen so that they are equal, maintaining the equality of Equation 14. In this configuration, due to the use of two sensors, the thermal effects are minimized.

In this case, by making all resistors with resistance equal to $R$, the strain-gauge $S_{3}$ equals $R-\Delta R$ and the strain-gauge $S_{4}$ equals $R+\Delta R$. Applying the equation 13 gives the equation 19 , which is manipulated similarly to the previous case to obtain the equation 20

$$
\begin{gathered}
V=-\frac{\Delta R}{2 R} V_{e x} \\
\frac{V}{V_{e x}}=-\frac{K \epsilon \times 10^{-3}}{2}
\end{gathered}
$$

In the complete bridge circuit four active strain-gauges are used, $S_{1}, S_{2}, S_{3}$ and $S_{4}$, according to Figure $3 \mathrm{c}$ In one typical assembly the strain-gauges $S_{2}$ and $S_{4}$ are arranged so that they are subjected to traction, $+\epsilon$, while the strain-gauges $S_{1}$ and $S_{3}$ are subjected to compression, - epsilon. In this configuration no resistors are used to complement the circuit and the thermal effects are minimized to the maximum.

In this case all the elements of the Wheatstone bridge are strain-gauges, considering the strain-gauges $S_{1}$ and $S_{3}$ with resistance $R-\Delta R$ and the strain-gauges $S_{2}$ and $S_{4}$ with resistance $R+\Delta R$, substituting these values in the equation 13 we obtain the Equation 21. By means of the manipulations already carried out, the equation 22 is obtained.

$$
\begin{gathered}
V=-\frac{\Delta R}{R} \\
\frac{V}{V_{e x}}=-K \epsilon \times 10^{-3}
\end{gathered}
$$

In this work, due to the educational approach of strain instrumentation, the quarter bridge circuit is used, which presents good results for the proposed purpose. For applications where greater precision and temperature compensation are required, it is recommended to use circuits such as half bridge or full bridge. For the implementation of the one-quarter bridge circuit of this work, $680 \mathrm{ohm}$ resistors were used and to balance the circuit out a 470 ohms trimpot was used and configured to zero the circuit output voltage.

\subsection{Data-acquisition}

The recent technological advances have contributed to the popularization and miniaturization of electronic devices. Among the microcontrollers popularized by users less experienced in programming and electronics, we can mention those of the PIC family and, more recently, the family of Arduino prototyping boards, based on the ATmega family of microcontrollers manufactured by Atmel. Arduino platforms stand out because they have their own programming language based on $\mathrm{C}++$, adapted to be simple and intuitive, and are marketed in the form of ready-to-use boards, which include the microprocessor and other components necessary for their use, eliminating the need for the user to create complex electronic circuits for the use of the microcontroller.

Due to its low cost, it has been discussed the use of Arduino microcontrollers in engineering applications that commonly involve instrumentation, monitoring of machines and structures and control of mechanical systems. In 22 the Arduino microcontroller was used along with low-cost sensors for vibration instrumentation. As in [11], the Arduino microcontroller is used for the instrumentation of vibrations in systems with various degrees of freedom by means of MEMS accelerometers. In [23] the Arduino was used with the wireless MEMS sensors in order to monitor the Himera viaduct in Italy.

In this work, it was utilized an Arduino Mega2560 R.3, Figure $4 \mathrm{a}$ and a Nanoshield LoadCell module, Figure 4b manufactured by Circuitar, based on the ADS1230 chip, which includes an amplifier, a filter and a 20-bit analog-todigital converter (ADC), which makes the module a highresolution tool for converting analog signals, especially 


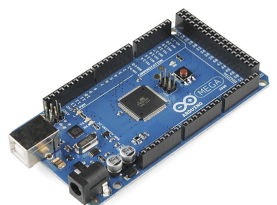

(a)

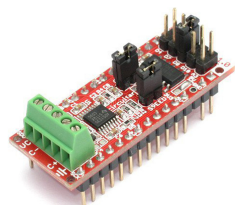

(b)

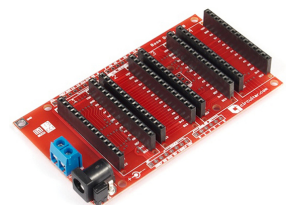

(c)
Figure 4: Boards utilized for data acquisition: 4a Arduino Mega2560 R.3, 4b Nanoshield Load Cell module and 4c Base Board.

those from load cells and Wheatstone Bridges, because these systems return low-amplitude signals. To facilitate the connection of the Arduino to the Nanoshield LoadCell module a Base Board, Figure $4 \mathrm{c}$ also manufactured by Circuitar was used, but this board is only used in order to facilitate the connection of one or more Nanoshield LoadCell modules. Although these modules have been used, others may also be used without any accuracy loss.

The Whetstone Bridge circuit implemented was powered with a $5 \mathrm{~V}$ DC voltage from the Arduino, while its signal outputs were connected to the amplification module mentioned before, where the amplification and discretization of the signal is performed. The choice of using an external ADC is be due to the resolution of most Arduino microcontrollers being 12 bits, thus the signal needs to be more amplified to have the same resolution as the Nanoshield LoadCell module, limiting the range of the data acquisition system. Figure 5 shows the connection diagram of the components used.

To program the Arduino assembly and the amplification module, it was utilized a library provided by the manufacturer of the Nanoshield Load Cell module, that can be found in its repository, 24, and to acquire the data an example sketch provided by the library was used.

\section{Experimental proccedures}

The bonding procedure of strain gauges must be thoroughly performed to avoid measurement errors, starting with the preparation of the surface for bonding. The surface where the strain-gauge will be glued must be carefully sanded using a fine water sandpaper with a granulometry of about 220 . First, the surface should be

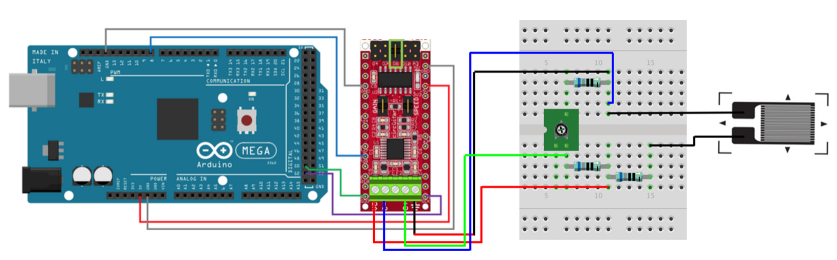

Figure 5: Connection diagram of the components for the data acquisition. sanded at $45^{\circ}$ of the center line of the strain-gauge, then sanded at $-45^{\circ}$ of the same line, so that the angle between the resulting lines of this process is $90^{\circ}$, forming an X. After this procedure, for educational purpose experiments, the bonding site must be cleaned with alcohol or acetone, but in applications that require more precision specific products should be used. After the cleaning, it is necessary to mark the bonding site, preferably using a pencil with fine graphite, so that there is no large graphite deposition in the place, which could end up affecting the measurement. The line where the deformation is to be measured and an auxiliary line at $90^{\circ}$, corresponding to the transverse sensitivity axis of the strain-gauge, need to be marked.

After the preparation process for the strain gauge bonding, the bonding process begins. For this, it is needed a glass base, previously cleaned with alcohol, tweezers, transparent tape and instant glue. To better handle the strain gauge with the tweezers, place it on the glass base so that its grid and solder terminals are up, note that the strain gauges should only be handled using the tweezers, avoiding any contact with the hands, reducing any risk of damage in them. Besides, the oiliness that is present in the hand can cause the oxidation of the strain-gauge grid. With the transparent tape glue the strain gauge onto the glass base. When removing the tape the strain gauge must be removed with the tape, still glued in it. The bonding of the strain-gauge to the tape is performed to facilitate the correct positioning and bonding of the transducer to the surface where the deformation will be measured. The tape and the strain gauge must be carefully positioned on the surface so that the previously marked lines match the strain gauge indicators. Figure 6 illustrates the correct positioning of the strain gauge. After the correct positioning, one must carefully peel off part of the tape until the strain gauge is raised to insert a small amount of instant glue under it, quickly reposition the tape as before and press the strain gauge so that bubbles do not form under it. Again, in applications where higher precision is required, it might be necessary to use specific products and more elaborate processes for bonding. After the cure time of the adhesive, carefully remove the tape and inspect the strain gauge with a magnifying glass. If the strain gauge has bubbles under its surface or if it is incorrectly positioned, remove it, discard it, and perform the preparation and gluing procedures again.

For the experiment of the crimped-supported shaft the marking of the reference line for the strain gauge was performed with the aid of a magnetic base, in which a pencil was fixed. With the shaft attached to the bench made for evaluation, a horizontal line was made in it with the aid of a mechanical marking gauge. The strain gauge on the shaft was carried out on the basis of the diagonal markings of the strain gauge, in this way the measurement of the strain occurs in a plane at $45^{\circ}$ from the cross section of the axis, which is the plane where the 


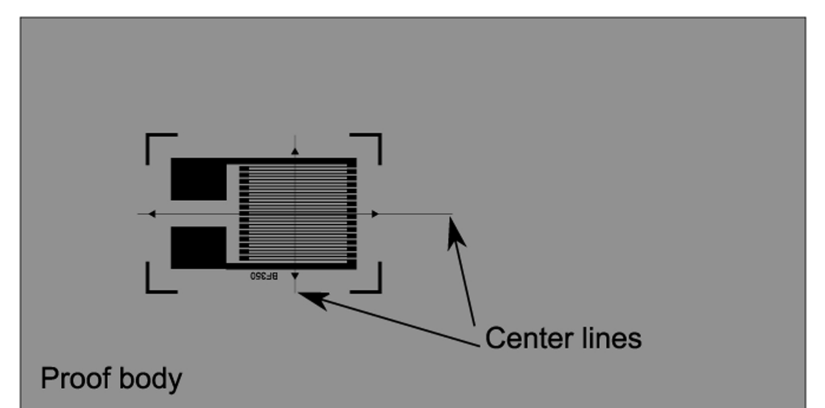

Figure 6: Correct positioning of the strain gauge at the measurement point.

main stresses occur. In experiments to measure torsion in shafts where good precision is required, a correct marking of the lines of the shaft must be performed, positioning the shaft horizontally, which can be done with the aid of a splitting device.

For the cantilever beam experiment a force of $0.981 \mathrm{~N}$ was applied, by means of positioning a block with known mass being at the free end of the beam, and the strain gauge was glued at $30 \mathrm{~mm}$ from the fixed end in the center of the upper face. In the torsion shaft experiment a torque of $3.6 \mathrm{~N} / \mathrm{m}$ was applied and the strain gauge was glued at $45^{\circ}$ of the axis of symmetry of the workpiece. In both experiments, 256 points were acquired.

The experiments were performed in two stages for each system. First, the force or torque are applied and, after a certain time, they were took off once. As in the second stage, the described process were performed twice, to analyze if the proposed measurement system has repeatability. The test benchs utilized for the experiments can be seen in Figure 7 and in Figure 8 the strain-gauges, already glued in the mechanical systems analyzed, are shown with more detail.

\section{Results and discussion}

Deformation measurements were performed in the two proposed systems, performing five measurements in each system for each case studied. In both systems, two cases were analyzed, in the first a momentary load was applied

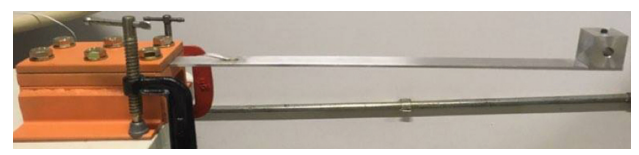

(a)

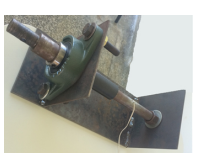

(b)
Figure 7: Test benches for experimental validation of the proposed measurement system: (7a) Cantilever beam and (7b) Crimped-supported shaft under torsion.

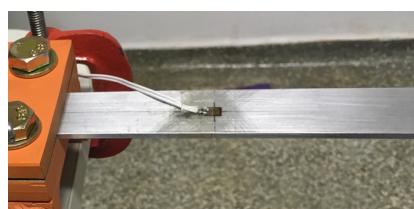

(a)

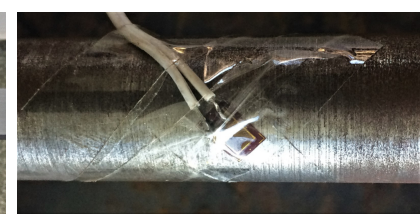

(b)
Figure 8: Strain-gauges glued to the analyzed mechanical system (8a) in experiment 1 and (8b) in experiment 2.

to the system and in the second case the same load was applied twice.

The signals were acquired with 256 points and sampling frequency of $80 \mathrm{~Hz}$, resulting around 3 seconds of data acquisition. In Figure 9 it is presented two strain signals in the time domain of the proposed system, where Figure 9a presents a signal for the first case studied and Figure $9 \mathrm{~b}$ for the second case.

As the deformation signals are obtained in the time domain and the load depend on the time, it is necessary to manipulate the measured signal to obtain the magnitude of the observed strain, for which the mean value of the deformation measured is considered as the resulting deformation on the proof body. In the first system, the cantilever beam, the deformation obtained in the experiment using the proposed method was $207.3 \mu \epsilon$, whereas in the second system, the crimped-supported shaft, the strain obtained was $13.3 \mu \epsilon$.

By means of the mathematical models presented in the section 2, a strain of $208.83 \mu \epsilon$ was obtained for the first system and of $14.02 \mu \epsilon$ for the second, while the results obtained through numerical simulation by finite elements resulted in $207.98 \mu \epsilon$ and $14.49 \mu \epsilon$, respectively for the first and second systems. For a better visualization of the results, they are presented in the Table 1.

The obtained results show that the proposed system has good efficacy for the proposed application, presenting satisfactory accuracy, because the results obtained were close to the analytical and numerical results, and presented low dispersion.

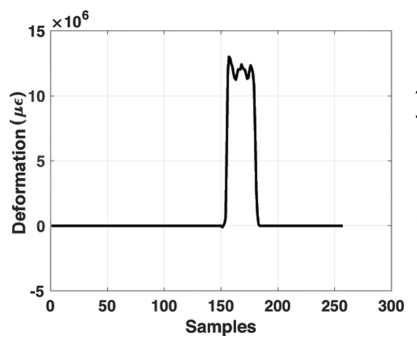

(a)

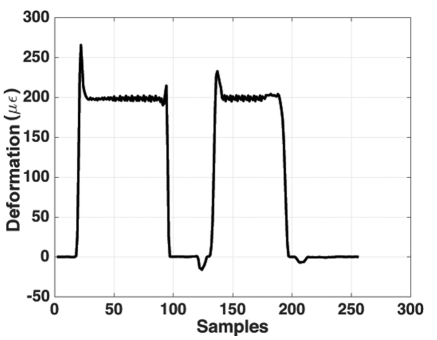

(b)
Figure 9: Samples of the time domain strain signals acquired: 9a First test, load applied once, and (9b) Second test, load applied twice. 
Table 1: Resultados obtidos.

\begin{tabular}{lcc}
\hline & Experimento 1 & Experimento 2 \\
\hline Análitico & 208.83 & 14.02 \\
Numérico & 207.98 & 14.49 \\
Experimental & 207.3 & 13.3 \\
\hline
\end{tabular}

\section{Conclusions}

In this work, it was proposed an alternative to commercial deformation instrumentation systems trough the Arduino microcontroller and low cost components. The main purpose of using low cost components is to allow undergraduate students who normally do not have contact with deformation instrumentation, due to the high cost of the equipment and the complexity of their operation, to do practical experiments that involve programming, electronics and instrumentation in a simple and intuitive way.

The proposed system proved to be reliable for the proposed purpose, since it presented good repeatability, there being no great dispersion between the samples, and it also presented good precision, obtaining values close to the numerical and analytical ones. The hardware used is of easy operation and its components are also well documented in the literature.

In future projects we intend to apply the methodology of measurement of deformation proposed to more complex systems, using different configurations of the Weatstone bridge and under different conditions of loads, as impact and dynamic loads.

\section{Acknowledgements}

The authors acknowledge the Conselho Nacional de Desenvolvimento Científico e Tecnológico ( $\mathrm{CNPq}$ ) for the support.

\section{References}

[1] F.P. Beer, E.R.Johnston Jr., J.T. Dewolf and D.F. Mazurek, Mechanics of Materials (McGraw-Hill, New York, 2006).

[2] D.B. Biggs and J.A. Shaw, In Behavior and Mechanics of Multifunctional Materials and Composites 2016 (International Society for Optics and Photonics, Las Vegas, 2016), p. 98000D.

[3] J.C. Butler, A.J. Vigliotti, F.W. Verdi and S.M. Walsh, Sensors and Actuators A: Physical 102, 61 (2002).

[4] D.J. Cohen, D.M. Mitra, K. Peterson and M.M. Maharbiz, Nano letters 12, 1821 (2012).

[5] L. Guerriero, G. Guerriero, G. Grelle, F.M. Guadagno and P. Revellino, Natural Hazards and Earth System Sciences 17, 881 (2017).

[6] E.S.B. Ismail, M.H. Habaebi and M.R. Islam, in 2017 IEEE 4th International Conference on Smart Instrumentation, Measurement and Application (ICSIMA),
(International Islamic University Malaysia, Putrajaya, 2017) p. 1.

[7] E. Jacquet, S. Joly, J. Chambert, K. Rekik and Patrick Sandoz, Skin Research and Technology 23, 531 (2017).

[8] L. Laloui, M. Nuth and L. Vulliet, International Journal for Numerical and Analytical Methods in Geomechanics 30, 763 (2006).

[9] K.H. Lim, C.M. Chew, P.C.Y. Chen, S. Jeyapalina, H.N. Ho, J.K. Rappel and B.H. Lim, Journal of biomechanics 41, 931 (2008).

[10] A. Lonsdale, Sensors-the Journal of Applied Sensing Technology 18, 51 (2001).

[11] M. Varanis, A.L. Silva and A.G. Mereles, Revista Brasileira de Ensino de Física 40, e1304 (2017).

[12] S. Wen and D.D.L. Chung, Cement and Concrete Research 31, 665 (2001).

[13] J. Wissman, A. Perez-Rosado, A. Edgerton, B.M. Levi, Z. Karakas, M. Kujawski, A. Philipps, N. Papavizas, D. Fallon, H. Bruck and E. Smela, Smart Materials and Structures, 22, 085031 (2013).

[14] E.P. Popov and T.A. Balan, Engineering mechanics of solids (Englewood Cliffs, Prentice Hall, 1990).

[15] S. Timoshenko, Strength of materials Part 1 (Krieger Publishing Company, Malabar, 1940).

[16] J.M. Gere and B.J. Goodno, Mechanics of Materials 5th (Brooks Cole, Boston, 2001).

[17] O.C. Zienkiewicz and R.L. Taylor, The finite element method for solid and structural mechanics (Elsevier, Amsterdã, 2005).

[18] K.J. Bathe, Finite element method (John Wiley \& Sons, New Jersey, 2008).

[19] T.J.R. Hughes, The finite element method: linear static and dynamic finite element analysis (Courier Corporation, Chelmsford, 2012).

[20] R.S. Figliola and D. Beasley, Theory and design for mechanical measurements (John Wiley \& Sons, New Jersey, 2015).

[21] J.P. Holman and W.J. Gadja, Experimental methods for engineers (McGraw-Hill, New York, 2001).

[22] M. Varanis, A.L. Silva, P.H.A. Brunetto and R.F. Gregolin, Revista Brasileira de Ensino de Física 38, 1301 (2016).

[23] F.L. Francesco, G. Navarra and M. Oliva, Meccanica 52, 3221 (2017).

[24] https://github.com/circuitar/Nanoshield_ LoadCell. 